\title{
POSSÍVEL TID GERADO NA REGIÃO POLAR E OBSERVADO SOBRE ESTAÇÕES EQUATORIAIS E DE BAIXAS LATITUDES
}

\author{
E. P. CAZUZA ${ }^{1 *}$, A. L. P. OLIVEIRA ${ }^{2}$, H. R. P. JÁCOME ${ }^{2}$, G. L. BORBA ${ }^{1}$, J. P. SILVA JUNIOR ${ }^{1}$, M. S. MARQUES ${ }^{1}$ \\ ${ }^{1}$ Universidade Federal do Rio Grande do Norte - UFRN, ${ }^{2}$ Instituto Federal do Rio Grande do Norte - IFRN \\ eliocazuza@ufrn.edu.br*
}

Submetido 19/03/2018 - Aceito 07/06/2018

DOI: $10.15628 /$ holos.2018.7108

\section{RESUMO}

Durante nossa pesquisa, detectamos e caracterizamos perturbações do tipo LSTID's (Large scale Travelling lonospheric Disturbances), em baixas latitudes, durante intensas tempestades geomagnéticas e também a forma como se propagaram. Utilizou-se registros ionosféricos obtidos a partir de digissondas do tipo CADI (Canadian Advanced Digital lonosonde) localizada na cidade de Natal e do tipo DSP (Digisonde Portable Souder) localizadas nas cidades de Cachoeira Paulista, Ramey e Eglin. Os dados de sondagem de Ramey e Eglin foram disponibilizados pela Global lonosphere Radio Observatory (GIRO). Para observar os efeitos de tempestades sobre a ionosfera polar, foram utilizadas imagens aurorais do polo Norte, obtidas pelo satélite POLAR, que mostraram a intensificação do eletrojato auroral pela injeção de partículas nas cúspides. O período analisado compreendeu os dias 06 e 07 de abril de 2000 e os índices utilizados para caracterizar a atividade magnética nesse período foram $\mathrm{Dst}, \mathrm{Kp}$, e $\mathrm{AE}$, além da componente $\mathrm{Bz}$ do campo magnético interplanetário. $\mathrm{A}$ tempestade ocorrida no dia 6 de abril apresentou um Dst $_{\text {min }}=-288 \mathrm{nT}$. As tempestades geomagnéticas são capazes de afetar o comportamento de todo o plasma ionosférico, afetando assim o funcionamento de estações de transmissão de sinal na faixa de telecomunicações e até a altitude de satélites que se encontram nessa região. A partir de gráficos de isolinhas foram verificados comportamentos anômalos da ionosfera sobre as quatro cidades, caracterizando a propagação como sendo do tipo LSTID's, gerados pela expansão do oval auroral nos polos.

PALAVRAS-CHAVE: LSTID'S, TEMPESTADES GEOMAGNÉTICAS, DIGISSONDAS.

\section{POSSIBLE TID GENERATED IN THE POLAR REGION AND OBSERVED ON EQUATORIAL STATIONS AND LOW LATITUDES}

\section{ABSTRACT}

During our research we detected and characterized LSTID (Large Scale Ionospheric Disturbances), in low latitudes, during intense geomagnetic storms and also the way they propagated. It was used ionospheric records obtained from digisondes of CADI type (Canadian Advanced Digital lonosonde) located in the city of Natal and DSP type (Digisonde Portable Souder) located in the cities of Cachoeira Paulista, Ramey and Eglin. The survey data from Ramey and Eglin were provided by Global lonosphere Radio Observatory (GIRO). To observe the effects of storms on the polar ionosphere, auroral images of the North pole, obtained by the POLAR satellite, were used, which showed the intensification of auroral electrojet by the injection of particles in the cusps. The
\end{abstract}

analyzed period included the days 6th and 7th, April 2000 , and the indices used to characterize the magnetic activity in this period were Dst, $\mathrm{Kp}$, and $\mathrm{AE}$, in addition to the $\mathrm{Bz}$ component of the interplanetary magnetic field. The storm on April 6th had a Dstmin $=-288$ nT. geomagnetic storm are capable of affecting the behavior of all ionospheric plasma, thus affecting the operation of signal transmission stations in the telecommunications band and the altitude of satellites in that region. Anomalous behavior of the ionosphere was verified on the four cities, characterizing the propagation as being of type LSTID's, generated by the expansion of the auroral oval at the poles.

KEYWORDS: LSTID'S, GEOMAGNETIC STORMS, DIGISONDES 


\section{INTRODUÇÃO}

A alta atmosfera terrestre, notadamente a faixa compreendida entre 60 e $1000 \mathrm{~km}$ de altura corresponde à camada denominada ionosfera que, se caracteriza como um magneto plasma fracamente ionizado e, como tal, é sujeito tanto a ação dos ventos neutros capazes de arrastar esse plasma provocando perturbações de grande e média escala, quanto a flutuações eletromagnéticas no geoespaço que circunda o planeta e que estão fortemente relacionados à atividade solar. Em ambas situações as perturbações podem afetar o plasma ionosférico produzindo ondas que podem se propagar por distâncias suficientemente grandes de modo a gerar irregularidades capazes de afetar a propagação de sinais de radiofrequência em um espectro que vai desde a faixa de $\mathrm{MHz}$, capazes de afetar as rádio comunicações, até $\mathrm{GHz}$, influenciando a precisão dos sistemas de posicionamento global, por exemplo o GPS.

Uma classe especial de perturbações, quase periódicas, que se propagam através do plasma ionosférico é a dos distúrbios ionosféricos propagantes (TID's - Travelling lonospheric Disturbances) cuja origem está relacionada à eventos ocorridos na atmosfera neutra tal como a propagação de ondas de gravidade (GW - Gravity Waves) e atinge a região $F$, provocando perturbações no perfil de densidade eletrônica do plasma ionosférico (HINES, 1974), como também à eventos relacionados com tempestades magnéticas e, nesse caso, as ondas de gravidade são geradas via aquecimento Joule devido à intensificação das correntes do eletrojato polar (BLUMEN, 1968), devido à deposição de partículas eletricamente carregadas e energia transportada pelo vento solar intensificado durante tempestades solares.

Perturbações ionosféricas oscilatórias, com características propagantes, tem sido observadas desde a década de 1940 e as primeiras tentativas de explicação para esse fenômeno através de um formalismo matemático foram dadas por Martyn (1950). A partir de 1960, uma série de pesquisas, tanto teóricas quanto experimentais, permitiu se estabelecer que essas perturbações eram causadas por ondas de gravidade, que se propagavam na atmosfera neutra por longas distâncias, afetando a distribuição eletrônica na ionosfera (HINES, 1960; FRANCIS, 1973; TESTUD, 1970; MACDOUGAL et al., 2011).

Ondas de gravidade são oscilações que ocorrem na atmosfera neutra devido às diferenças de pressão geradas por aquecimentos anisotrópicos da atmosfera e pela força da gravidade (HINES, 1960; TSUDA et al., 2000). Essas ondas, com diversas escalas de comprimento de onda e período, são geradas principalmente na baixa e na média atmosfera e se propagam pela atmosfera com amplitudes crescentes com a altura (VARGAS, 2007; OLIVEIRA, 2016). As ondas de gravidade representam um importante mecanismo de dissipação da energia contida na atmosfera ou nela injetada durante tempestades magnéticas. No Quadro 1 pode-se observar os períodos característicos das ondas de gravidade em comparação com os principais períodos de outros tipos de ondas atmosféricas.

A ocorrência de ondas de gravidade na ionosfera terrestre causa, entre outros fenômenos, significativas variações de densidade eletrônica na região $F$, efeito amplamente estudado devido ao seu importante papel no transporte de momentum e energia através da atmosfera (HINES, 
1960; BOWMAN, 1965; THOME, 1968; BEER, 1974; HEDIN, 1987; MAYR et al., 1990; MCLANDRESS, 1998).

Quadro 1 - Principais tipos de ondas Atmosféricas, adaptado de Beer (1974).

\begin{tabular}{|l|c|c|}
\hline \multicolumn{1}{|c|}{ Ondas } & Período & Importância \\
\hline Acústicas & $\leq 5$ minutos & Troposfera \\
\hline Gravidade & 5 minutos -3 horas & Ionosfera \\
\hline Marés & $24 /$ m horas $(m=1,2, \ldots)$ & Variações Geomagnéticas \\
\hline Roosby & $\geq 12$ horas & Meteorologia \\
\hline
\end{tabular}

Apesar de ser um fenômeno típico da atmosfera neutra, as ondas de gravidade são um ponto de conexão entre a meteorologia e a física da ionosfera, ou seja, entre eventos que ocorrem na troposfera e são acoplados com a ionosfera. Essas perturbações possuem fontes diversas tais como colisões de frentes de pressão, erupções vulcânicas e terremotos (WRASSE, 2004; KAFLE, 2009), além do aquecimento Joule, na alta atmosfera, das regiões aurorais durante tempestades magnéticas, causado pelo aumento do fluxo de partículas que atingem a atmosfera e que provocam perturbações que se propagam no sentido dos polos para o equador (TESTUD, 1970), fenômeno responsável pelos TID's de larga escala.

Neste sentido, as interações entre as camadas, ou acoplamentos, estão relacionados de um lado pela atividade solar, e de outro pela dinâmica da própria troposfera devido a sua capacidade de propagar ondas mecânicas nos mais diversos comprimentos de onda. Um exemplo de destaque na literatura, como destaca Bishop et al. (2006), são as tempestades tropicais e tornados que provocam acoplamento entre a ionosfera/termosfera e a troposfera. Tais eventos são capazes de gerar ondas de gravidade, as quais podem atingir a alta atmosfera se propagando na ionosfera na forma de TID's.

A partir da década de 1990, com as pesquisas tentando obter melhor entendimento dos mecanismos de acoplamento entre os sistemas troposfera-mesosfera-termosfera, surgiram muitos trabalhos descrevendo essas interações, assim como modelos de acoplamento para previsões, os quais mostram concordância com conjuntos de dados observacionais (REES, 1995; HOFFMANN et al., 2012 e YIĞIT et al., 2016).

Buscando ampliar o entendimento acerca do fenômeno acima descrito, nesta pesquisa discute-se o evento ocorrido no mês de abril de 2000, que representa uma rara oportunidade de mapeamento do comportamento da ionosfera sobre o setor sul-americano de latitudes de tal modo que foi possível verificar a propagação de ondas desde o instante da expansão auroral até os TID's sobre algumas estações situadas ao longo do seu percurso dos TID's.

Eventos que ocorrem no espaço interplanetário podem repercutir sobre o planeta como eventos extremos, como é o caso da tempestade magnética de 1989 , considerado o evento mais intenso das últimas dezenas de anos. Esta intensa tempestade induziu correntes no solo, causando 
um blackout (ou apagão) na cidade de Quebec, no Canadá, e ainda provocou alterações na órbita e interrupção da operação de satélites. Cientistas tem alertado a sociedade para os efeitos de eventos extremos que ocorrem no Sol e seus efeitos sobre a Terra (PINHEIROS, 2012), o que, em parte, justifica os estudos de tais fenômenos atmosféricos.

Neste contexto, o presente artigo tem como objetivo analisar os TID's de larga escala sobre as cidades de Cachoeira Paulista, Natal, Ramey e Eglin durante a tempestade magnética ocorrida em 06 de abril de 2000, utilizando registros ionosféricos de digissondas do tipo CADI (Canadian Advanced Digital lonosonde), localizada em Natal, e do tipo DSP (Digisonde Portable Souder), localizadas em Cachoeira Paulista, Ramey e Eglin. No estudo dos efeitos dessa tempestade sobre a ionosfera polar foram utilizadas imagens aurorais do polo Norte, obtidas pelo satélite POLAR.

\section{REVISÃO BIBLIOGRÁFICA}

Os distúrbios ionosféricos propagantes, os TID's (Travelling lonospheric Disturbances), são irregularidades periódicas no plasma ionosférico movendo-se com velocidades da ordem de dezenas até algumas centenas de quilômetros por hora. Eles têm sido observados movendo-se por distâncias horizontais de milhares de quilômetros em regiões de médias latitudes e acredita-se que seja um fenômeno global (RISHBETH e GARRIOTT, 1969). São comumente distribuídos em duas classes: os distúrbios ionosféricos de média escala (MSTID's - Medium Scale TID's) e os de larga escala (LSTID's - Large scale TID's) (RIEGER, 2002).

Os TID's de larga escala possuem períodos compreendidos entre 30 minutos a 3 horas e sua onda constituinte, muitas vezes, apresenta um caráter oscilatório, compreendendo vários ciclos (FRANCIS, 1975). Seu comprimento de onda é igual ou superior a $1000 \mathrm{~km}$, apresentando velocidades de fase cujas variações são compreendidas entre $300-1000 \mathrm{~m} / \mathrm{s}$ (STEPANOV, 2012), suas possíveis fontes são tempestades geomagnéticas, que aquecem a termosfera por efeito Joule, nas regiões polares como destaca Cândido (2008) e Cazuza (2014), e possuem a direção de propagação no sentido polo-equador (HARGREAVES, 1992). Os LSTID's são manifestações ionosféricas das ondas de gravidade (HAJKOWICZ, 1990; IDRUS, 2013).

Hawlitschka (2006) investigou os LSTID's em diferentes condições geomagnéticas, utilizando medidas de alta frequência e identificou que ocorrem aproximadamente 2 horas após o crescimento das tempestades magnéticas, podendo ocorrer à noite quando há condições geomagnéticas calmas.

O meio interplanetário do Sistema Solar é varrido continuamente pelo vento solar, que, quando perturbado pelo aumento da atividade solar, pode causar mudanças na geometria do campo geomagnético e nas correntes elétricas que fluem na alta atmosfera terrestre, o que leva a variações na sua intensidade (SILVA JÚNIOR, 2015). Na Figura 1, pode-se observar uma representação da influência do vento solar no campo geomagnético da terra, nela pode-se notar compressão em um dos lados da magnetosfera, e expansão no lado oposto. No lado da compressão, chamado de diurno, a magnetosfera se estende por uma distância de aproximadamente 10 raios terrestres, enquanto que, no lado oposto (noturno), por 
aproximadamente 60 raios terrestres (RISHBETH e GARRIOTT, 1969), caracterizando a longa cauda magnética, chamada de cauda magnetosférica. Esse formato é devido ao arraste contínuo do campo terrestre pelo vento solar na direção do seu deslocamento.

Tempestades geomagnéticas são grandes perturbações no campo magnético terrestre causadas por eventos solares intensos tal como erupções solares, que geram alterações na velocidade e na densidade do vento solar (DAL POZ e CAMARGO, 2006). A intensidade de uma tempestade geomagnética é determinada pela atenuação sofrida pelo campo geomagnético devido às correntes de anel ${ }^{1}$. Essa variação é mostrada pelo índice magnético Dst (storm time disturbance), baseado na variação do campo magnético da Terra. Entre os índices geomagnéticos, os três mais utilizados são Dst, Kp e AE, como destacado no trabalho de Nogueira (2009).

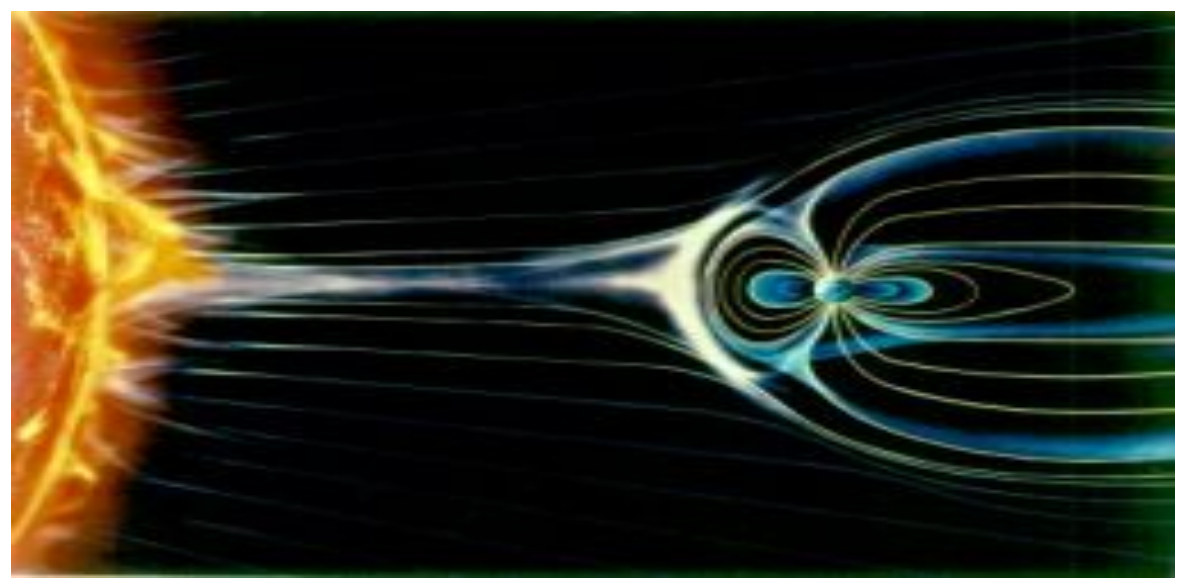

Figura 1 - influência do vento solar no campo geomagnético. Fonte: <https://www.3bmeteo.com/giornalemeteo/tempesta-magnetica-investe-la-terra--cosa-ci-dice-l--indice-k-planetario--62005> Acesso em novembro de 2017

O Dst é medido em unidades de nano tesla (nT) e tem uma resolução temporal de uma hora. Esse parâmetro representa principalmente a intensidade da corrente de anel nos períodos perturbados (YAMASHITA, 1999). A figura 2 mostra a curva do índice Dst obtida entre os dias 5-8 de setembro de 1982, quando ocorreu uma intensa tempestade geomagnética, com suas fases características. A partir do índice Dst, é possível observar que o início de uma tempestade magnética é acentuado por um pico de intensidade conhecido como fase inicial ou início súbito (sudden storm commencement), o qual ocorre logo após as dezoito horas do dia 05.

Logo após o pico inicial, ocorre a fase principal (main phase) da tempestade e é caracterizada por uma brusca queda no valor do índice Dst, devido à intensificação da corrente de anel que flui na magnetosfera, fase essa que pode levar até algumas horas. A próxima fase característica de uma tempestade magnética é a fase de recuperação (recovery phase), que pode durar até alguns dias, ela se inicia logo após o índice alcançar o mínimo valor e então ele volta a

\footnotetext{
${ }^{1}$ Corrente de anel: As correntes de anel são geradas pelo movimento de partículas energéticas presas às linhas do campo magnético terrestre numa altitude entre 4 e 6 raios terrestres. Tais partículas se movimentam em sentido horário, observando-se sobre o polo norte, gerando um campo magnético contrário ao campo terrestre (Hargreaves, 1992).
} 
subir até atingir valores aproximados ao de períodos calmos, ou seja, quando não há tempestades (YAMASHITA, 1999).

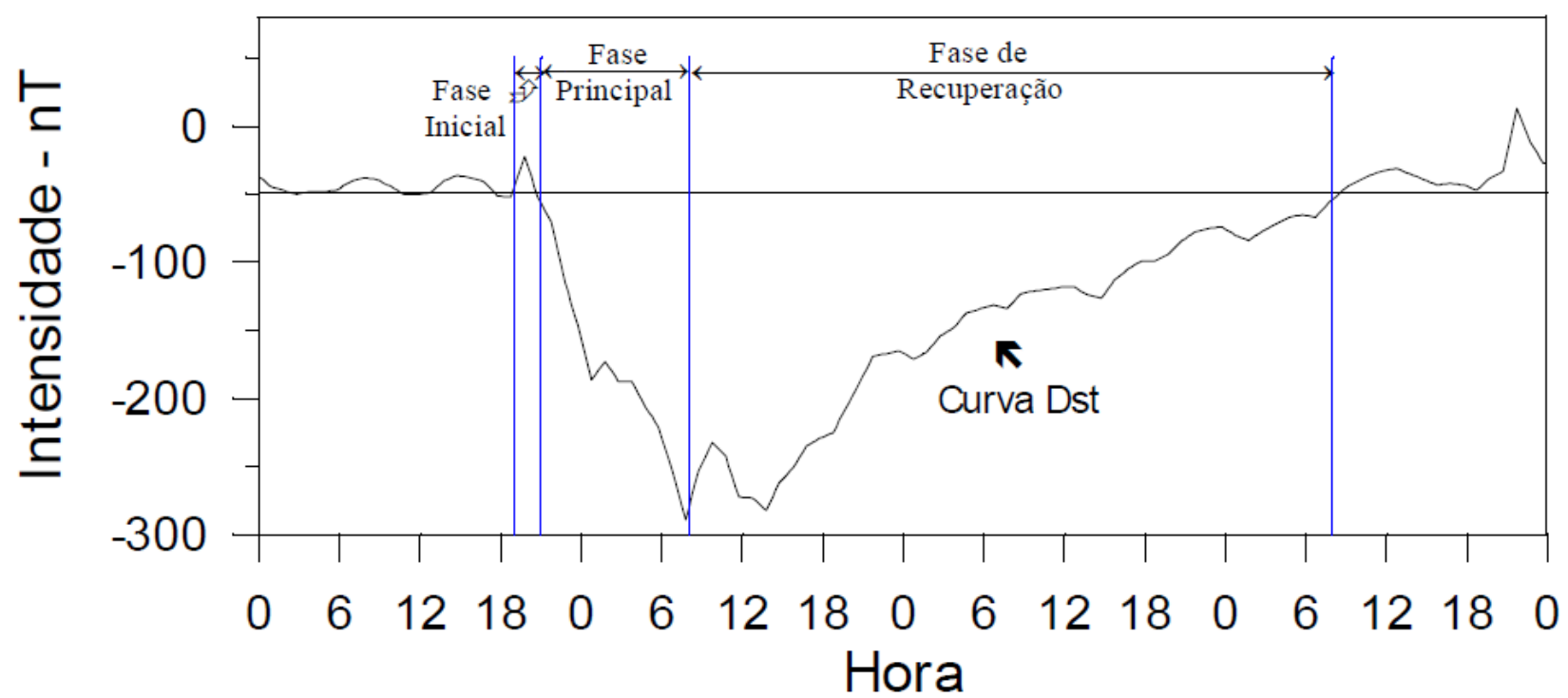

Figura 2 - Curva Dst entre os dias 5-8 de setembro de 1982. Fonte: Yamashita (1999)

O índice planetário Kp está relacionado ao grau de perturbação do campo magnético terrestre, fornecendo uma média quantitativa do nível de perturbação planetária. É dado em parâmetros de uma escala logarítmica e contém variações ocorridas na atividade magnética em intervalos de três em três horas, relativas à curva do dia geomagneticamente calmo para um determinado observatório magnético (FEDRIZZI, 2003).

$O$ índice do eletrojato auroral AE se assemelha ao índice Dst por representar a variação da componente $\mathrm{Bz}$ do campo magnético. Entretanto, esse índice é obtido em intervalos de apenas um minuto a partir de dados de 12 estações distribuídas ao longo da região auroral do hemisfério norte.

\section{METODOLOGIA}

Os LSTID's podem ser observados através de várias técnicas de medição, tais como utilização de ionossondas (HAJKOWICZ et al., 1991; AFRAIMOVICH et al., 2008), radar de espalhamento incoerente (STERLING et al., 1971; NICOLLS et al., 2004), airglow (SOBRAL et al., 1985; SHIOKAWA et al., 2002), digissondas (STEPANOV et al., 2012), Sistema de Posicionamento Global (AFRAIMOVICH et al., 2000; 2008; NGWIRA et al., 2012) e, recentemente, muitos estudos relatam o uso de várias técnicas juntas tais como ionossondas, digissondas e GPS (LYNN, 2008).

Nesta pesquisa, para a análise de ionogramas e elaboração de gráficos de isolinhas (altura versus frequência), proporcionando a altura em função da frequência, utilizou-se dados de ionossonda do tipo CADI instalada no Centro de Lançamento da Barreira do Inferno (CLBI), na cidade de Natal-RN, e dados de ionossondas do tipo DPS, localizadas na cidade de Cachoeira Paulista-SP, assim como dados de Ramey e Eglin, fornecidos pelo GIRO (Global lonosphere Radio Observatory). Após o contato com os responsáveis pelos dados através do site 
http://giro.uml.edu/, é gerada uma senha e login, para que o usuário tenha acesso aos dados de sondagem. Para observar os efeitos dessa tempestade sobre a ionosfera polar, foram utilizadas imagens aurorais do polo Norte, obtidas pelo satélite POLAR, através do site https://cdaweb.sci.gsfc.nasa.gov/index.html/. Na Figura 3 pode-se observar a representação das posições das estações de sondagem ionosférica de Eglin (A), Ramey (B), Natal (C) e Cachoeira Paulista (D).

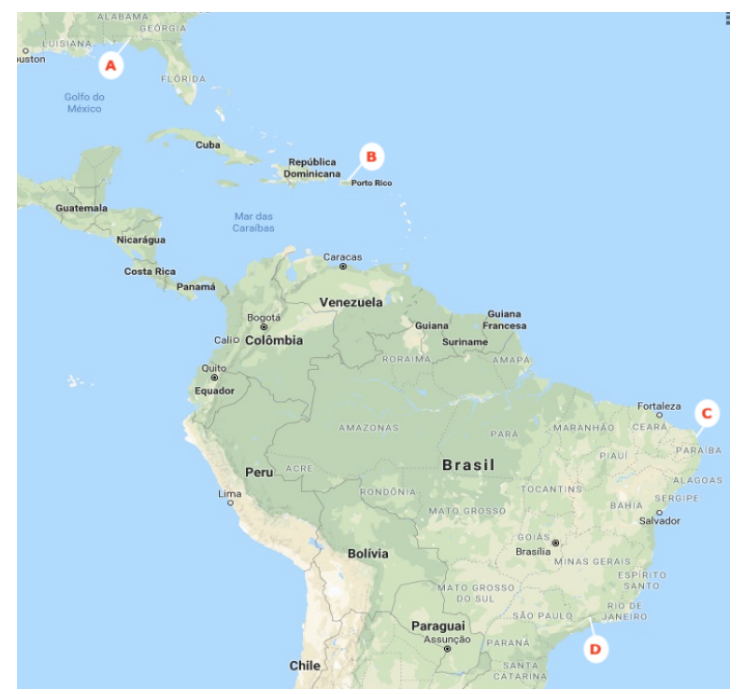

Figura 3: Figura ilustrativa das posições das estações de sondagem ionosférica de Eglin (A), Ramey (B), Natal (C) e Cachoeira Paulista (D). Retirada do Google maps em março 2018.

Com base nas discussões aqui estabelecidas, as intensas tempestades magnéticas, aquelas que possuem índice $\mathrm{Kp}>5$, são fontes das ocorrências das perturbações ionosféricas de larga escala. Assim, foi realizado um estudo de caso correspondente aos dias 06 e 07 de abril de 2000, em que foram detectadas tempestades com essas características, cuja variação da intensidade do campo geomagnético chegou a $-288 \mathrm{nT}$.

O mês de abril de 2000 se caracterizou como um período de intensa atividade solar, e as tempestades desse período tem sido intensamente estudadas (HUTTUNEN et al., 2002; DAL LAGO et al., 2004). No evento do dia 06 de abril, observou-se uma intensa ejeção de massa coronal seguidas por fortes tempestades geomagnéticas, detectadas por magnetômetros instalados em satélites e na superfície terrestre.

$\mathrm{Na}$ análise dos parâmetros da perturbação necessários para estudo, tais como amplitude $(A)$, período $(T)$, comprimento de onda vertical $\left(\lambda_{z}\right)$ e velocidade vertical de fase $\left(v_{z}\right)$, foi adotado o método utilizado por Fagundes (2007), no qual o período é determinado a partir da média dos períodos encontrados para um conjunto de frequências e a velocidade de fase vertical é determinada pelo deslocamento em altitude dos máximos das ondas sobre o respectivo deslocamento em tempo do mesmo ponto. O mesmo procedimento é adotado para a determinação do deslocamento de tempo. Sabendo os resultados do deslocamento em altura e tempo é possível então calcular a velocidade de fase vertical. 


\section{RESULTADOS E DISCUSSÃO}

Segundo Fagundes (2007), é possível observar ondas de gravidade utilizando a técnica de rádio-sondagem desde que se tenha uma boa resolução temporal, o que corrobora com os resultados que serão apresentados, uma vez que foi possível visualizar e estudar esse fenômeno sobre regiões de baixas latitudes durante períodos geomagneticamente perturbados, ou seja, durante intensas tempestades magnéticas.

A tempestade estudada ocorreu de forma intensa no dia 06 de abril de 2000 e sua fase de recuperação durou alguns dias. Ela foi selecionada para ter seus efeitos ionosféricos estudados, notadamente por sua grande intensidade, que contribui para a identificação de LSTID’s.

Nesta seção, os índices referentes ao comportamento do campo magnético serão apresentados e analisados com a finalidade de identificar e classificar os eventos ocorridos nos dias 06 e 07 de abril de 2000. A Figura 4 representa uma série de índices (Bz, Dst, Kp e $A E$ ) correlacionados ao período de 48 horas, correspondentes aos dias estudados.

O evento do dia 06 é classificado como tempestade muito intensa (Dst $\operatorname{mín}=-288 \mathrm{nT}$ ). $\mathrm{Na}$ tempestade, a fase principal teve duração de aproximadamente 7 horas, onde o início súbito ocorreu por volta das 16h40min (UT), Horvath e Lovell (2013), o que nos dá aproximadamente 07h50min antes do início das oscilações sobre Cachoeira Paulista.

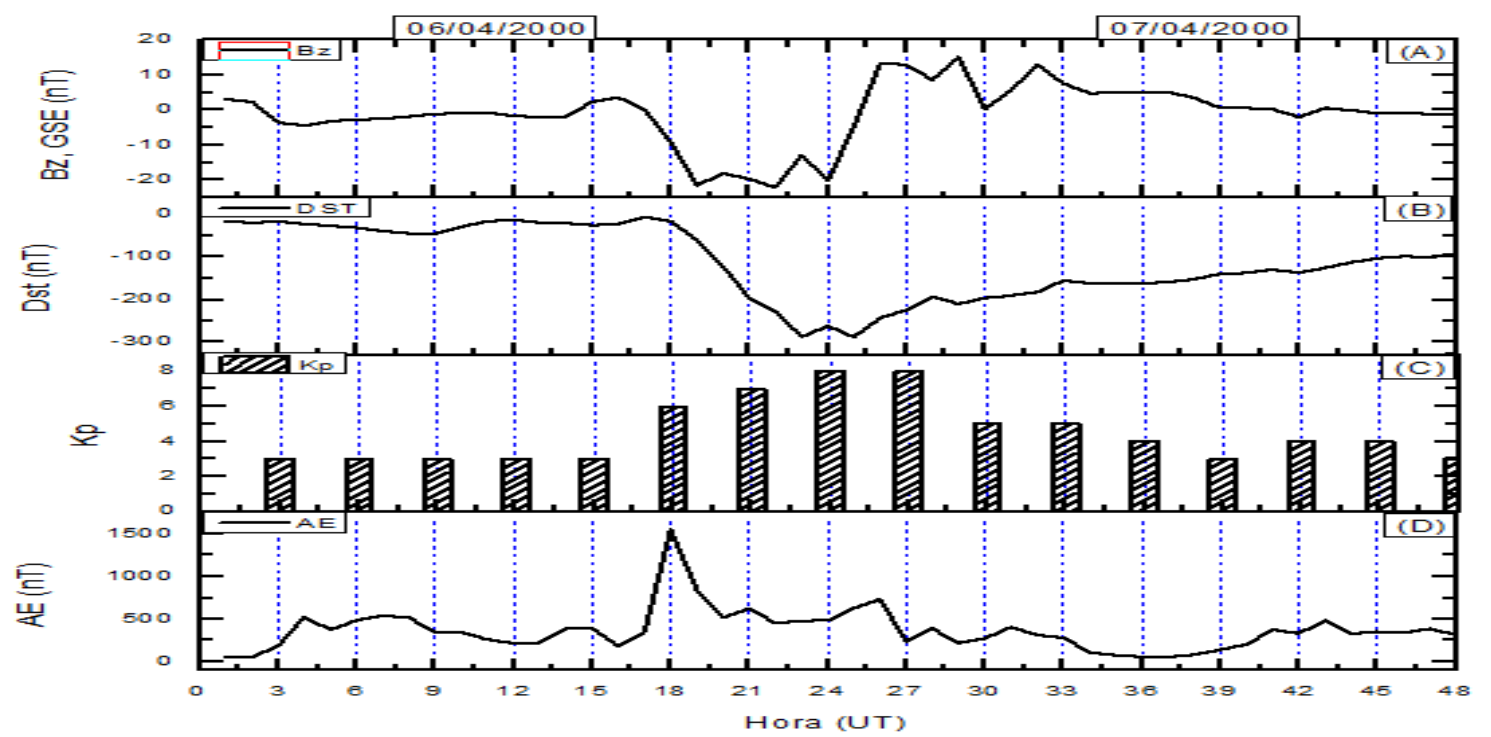

Figura 4: Dados horários do campo magnético (Bz) e dos índices Dst, Kp, e AE para os dias 06 e 07 de abril de 2000.

A fase inicial das tempestades é caracterizada pelo aumento abrupto do índice Dst devido à compressão das linhas do campo terrestre por choque, porém, a partir da análise dos dados de Dst, como mostra a Figura 4 (B), foi analisado que não houve aumento considerável, ou seja, não foi observado o início, uma vez que o maior crescimento observado foi de apenas 16 nT em um intervalo de 1 hora, entretanto fica clara a fase principal da tempestade, seguida pela fase de recuperação.

Quanto ao sentido do campo magnético interplanetário, durante a tempestade, pôde-se observar que a componente $\mathrm{Bz}$ obteve valores negativos, indicando seu sentido Norte-Sul, 
antiparalelo ao campo geomagnético. $\mathrm{O}$ índice $\mathrm{Kp}$ alcançou valores superiores a 8 , caracterizando a atividade magnética como de tempestade intensa. Verifica-se ainda, pelo índice $A E$, uma intensificação das correntes do eletrojato auroral.

\subsection{Efeitos na ionosfera polar}

Grandes tempestades geomagnéticas geram na camada $\mathrm{E}$ da ionosfera polar uma intensificação das correntes do eletrojato auroral. Essa intensificação pode ser indicada pelo aumento do índice geomagnético AE como também pelo aumento na densidade de partículas nas regiões polares. Portanto, além dos valores horários de $A E$, apresentados na Figura 4 (D), também foram utilizadas imagens feitas pelo experimento UVI (Ultraviolet Imager), no satélite POLAR, para se observar esses efeitos na ionosfera.

Na Figura 5, a imagem representa o estado normal da região auroral, com a região em azul mais claro indicando o lado diurno da Terra. Entretanto, a partir da imagem 5B, referente às 16:39 UT, nota-se um pequeno aumento de densidade no lado noturno quando, possivelmente, ocorre o início da tempestade magnética. Tal aumento no lado noturno pode ser consequência de uma rápida reconexão magnética entre as linhas do CMI (Campo Magnético Interplanetário) e do campo magnético terrestre na cúspide, no lado noturno (FUSELIER et al., 2000).

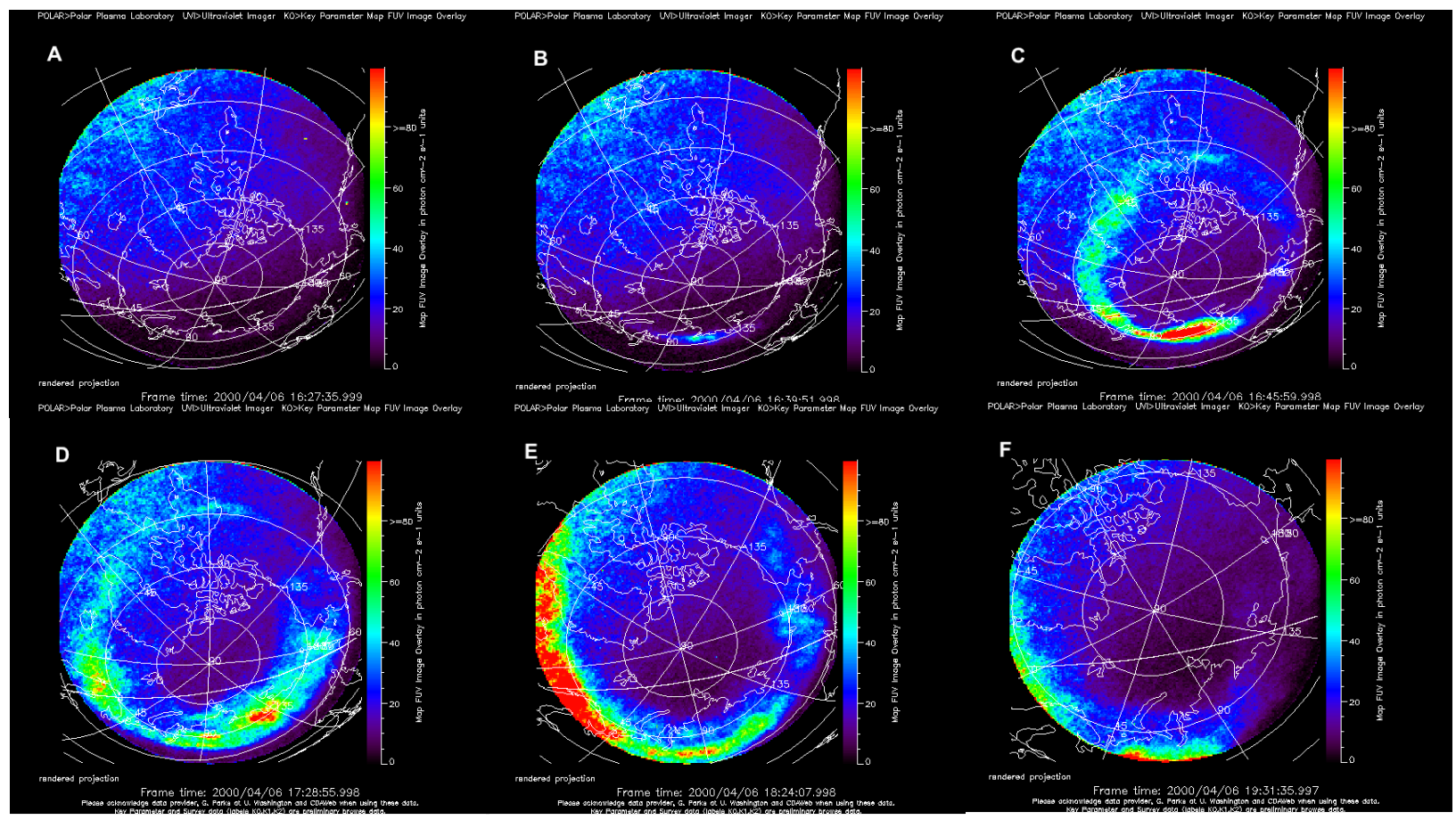

Figura 5: Imagens de ultravioleta distante obtidas pelo experimento UVI, do satélite POLAR, mostrando a densidade de prótons na região autoral Norte. Retiradas de https://cdaweb.sci.gsfc.nasa.gov/cgibin/eval2.cgi. Acesso em novembro de 2017.

Em seguida, 6 minutos depois, às 16:45 UT, como mostra a imagem 5C, a densidade de prótons apresentou um aumento significativo ao longo de todo o oval auroral. Esse aumento abrupto coincide com o aumento do índice $A E$, que passa de $350 \mathrm{nT}$ a $1550 \mathrm{nT}$ no horário 
compreendido entre $16-17$ horas (UT). Da imagem 5C em diante, observa-se a expansão do oval auroral em direção às latitudes mais baixas, representado pela migração das cores quentes para as extremidades das imagens. Essa migração se dá devido ao gradiente de temperatura gerado por efeito Joule nos polos devido à intensificação das correntes ionosféricas, transmitindo os efeitos da tempestade a regiões de latitude mais baixa.

\subsection{Efeitos na ionosfera equatorial}

Um dos efeitos da tempestade geomagnética na ionosfera equatorial está relacionado à transferência de energia da região auroral através das TIDs e pode ser observado pela elevação da camada ou aumento da densidade eletrônica (GUO et al., 2014).

Tomando por base um período geomagneticamente perturbado, foram selecionados dados de altura versus frequência, fornecidos pelas digissondas localizadas em Eglin, Ramey, Natal e Cachoeira Paulista, possibilitando-se a construção de gráficos de isolinhas para esse período. As Figuras 6A, 6B, 6C e 6D correspondem, respectivamente, as isolinhas para os dias 6 e 7 de Abril de 2000, podendo-se observar um comportamento oscilatório do plasma ionosférico, sobre as estações, identificando uma assinatura típica de um LSTID's.

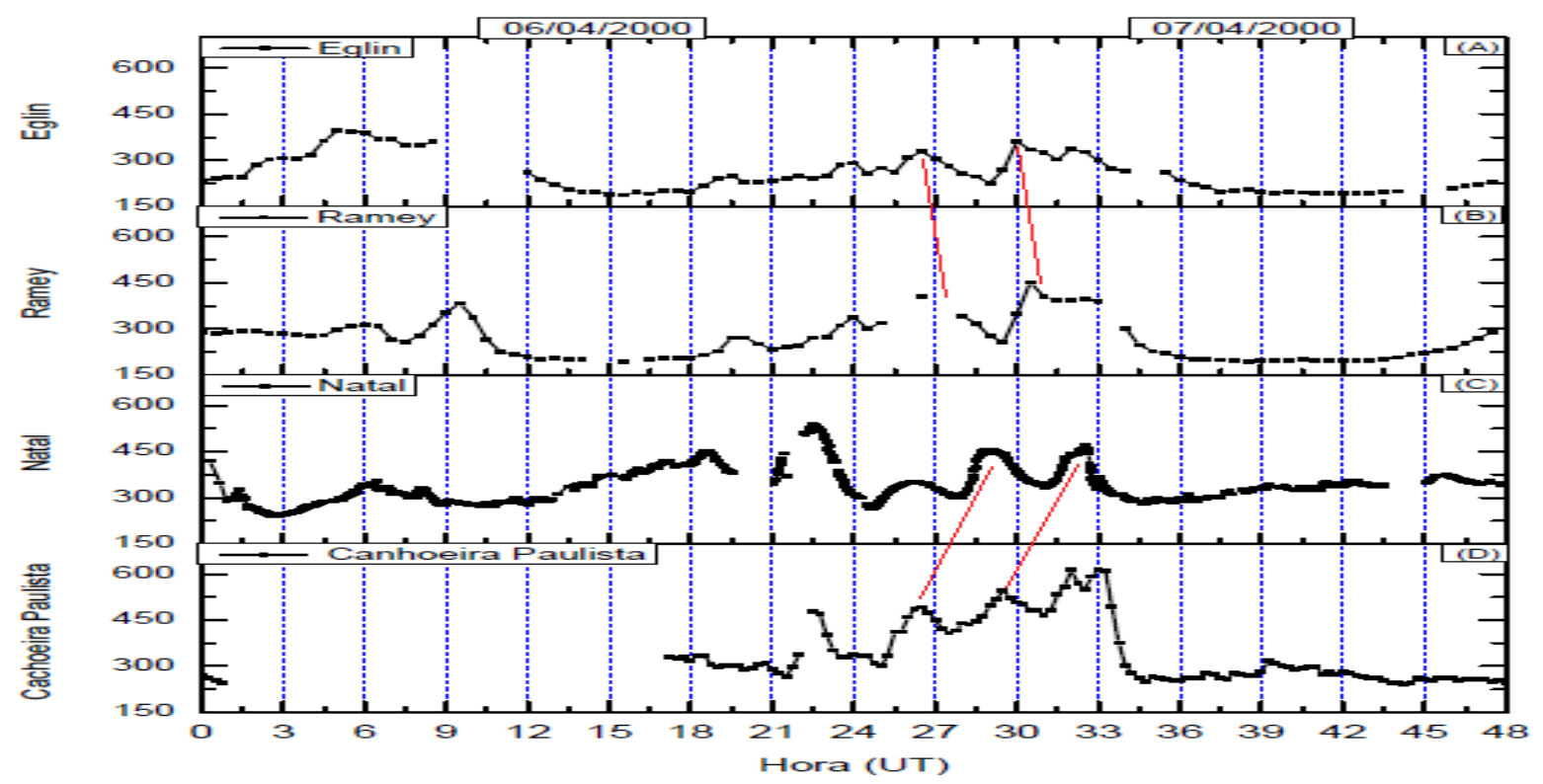

Figura 6: Curvas de isodensidade sobre as cidades de Eglin, Ramey, Natal e Cachoeira Paulista, para os dias 6 e 7 de Abril de 2000.

Ainda da Figura 6, verifica-se que a perturbação aparece primeiro nos registros de Cachoeira Paulista e só depois nos de Natal, assim como, aparece primeiro nos registros de Eglin e depois no de Ramey, o que permite concluir que a perturbação foi gerada ao sul de Cachoeira Paulista e ao norte de Eglin, passando sobre essas cidades e se propagando em direção ao equador, em seguida sobre Natal e Ramey. Além disso, pode-se observar que a amplitude da perturbação diminui à medida em que se dirige ao equador, sugerindo que sua propagação seja ao longo da linha do campo geomagnético. 
Na tentativa de associar tal perturbação à expansão do oval auroral, como destacado na Figura 5, devido à tempestade geomagnética ocorrida no dia 6, foi estimada a velocidade de propagação dessa expansão. Para tanto, foi considerado que o efeito da tempestade sobre os polos se dá de forma simétrica, ou seja, que o que foi observado no polo Norte pelas imagens aurorais pode ser extrapolado para o polo Sul. Além disso, considerou-se que a expansão nos polos teve início às $16 \mathrm{~h} 40 \mathrm{~min}$ (UT) e chegou sobre Natal às $2 \mathrm{~h} 00 \mathrm{~min}$ (UT) do dia 7 , a velocidade encontrada foi de, aproximadamente, $250 \mathrm{~m} / \mathrm{s}$, resultado que está de acordo com a ordem de grandeza das velocidades médias de TID's mostradas nos trabalhos de Hines (1960), Hunsucker (1982) e Vlasov et al. (2011). Portanto, infere-se neste trabalho que as perturbações observadas na ionosfera equatorial sobre as regiões estudadas são consequência dos TID's gerados pela injeção de partículas nas cúspides, no dia 06 de abril. Para Natal, essa oscilação apresentou um período da ordem da 03h00min e amplitude superior a $100 \mathrm{~km}$. O LSTID começou a se formar por volta das 03 h00min (UT) do dia 07 de abril de 2000 e seu término ocorreu por volta das 09h00min (UT) do mesmo dia.
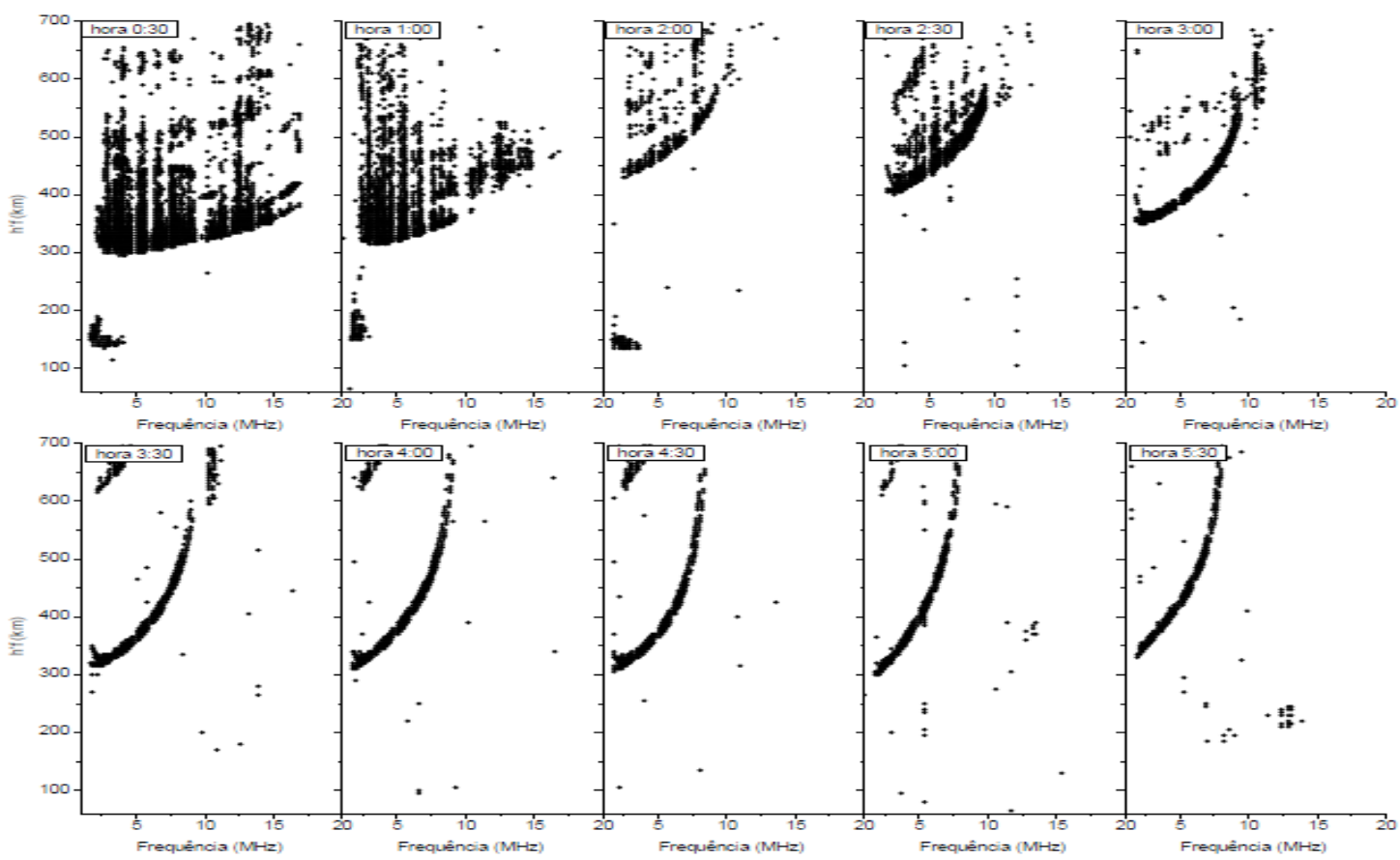

Figura 7: Sequencia de ionogramas mostrando a evolução temporal durante a propagação de ondas de gravidade entre 14:20 UT (11:20 LT) e 15:05 UT (12:05LT) de 07 de abril de 2000, observado em São José dos Campos

$\left(23^{\circ} 10^{\prime} \mathrm{S} ; 45^{\circ} 53^{\prime} \mathrm{O}\right)$.

Outra forma de observar a perturbação aqui estudada é a partir da sequência de ionogramas destacados na Figura 7, que representam o comportamento ionosférico referente ao dia 07 de abril de 2000 para a cidade de Cachoeira Paulista. Pode-se observar, ainda, o deslocamento vertical, para cima e para baixo, do plasma ionosférico durante a passagem do TID. É viável concluir que não só foi possível observar esse fenômeno utilizando ionossondas, como também caracterizá-lo e determinar seus principais parâmetros. 
Outro efeito ionosférico de tempestades geomagnéticas que pode ser observado em ionogramas é o black out, que consiste no "desaparecimento" da ionosfera nos registros de ionossonda. Acredita-se que tal fenômeno pode estar associado a duas causas distintas: a deriva vertical da camada F ou o intenso aumento de densidade eletrônica na camada D.

\section{CONCLUSÕES}

Neste trabalho, foram estudados os efeitos da tempestade geomagnética ocorrida no dia 06 de abril de 2000 sobre a ionosfera polar e equatorial. Ela foi classificada como muito intensa a partir dos valores do índice Dst e seus efeitos foram analisados sobre a ionosfera nas regiões polar e equatorial.

A ionosfera polar foi representada por imagens de ultravioleta distante das emissões aurorais no polo Norte. Tais imagens indicaram o início de injeção de partículas pelas cúspides através do aumento de densidade de prótons a partir das 16:45 UT do dia 06 de abril de 2000, seguido da expansão do oval auroral em direção às baixas latitudes. Já a ionosfera equatorial foi representada por gráficos de isodensidade referentes às estações de sondagem ionosférica localizadas em Cachoeira Paulista, Natal, Ramey e Eglin. A partir desses gráficos, foi possível reconhecer, no dia 07, perturbações ionosféricas sobre essas cidades.

As velocidades de propagação e amplitudes dos eventos estudados estão de acordo com os resultados que constam na literatura voltada para o fenômeno, garantindo a confiabilidade nos tratamentos de dados e nos equipamentos utilizados. Foi observado que os parâmetros de onda (período $\mathrm{T}$, comprimento de onda vertical vz, velocidade de fase vertical $\mathrm{Vz}$ e velocidade de propagação), estão dentro do esperado pela na literatura, ou seja, períodos superiores a trinta minutos e velocidade de propagação de centenas de quilômetros por hora. Neste sentido, se destaca como uma vantagem o uso das imagens aurorais, tendo em vista o curto intervalo de tempo entre cada registro, permitindo observar variações do eletrojato autoral de forma mais precisa.

Além disso, foram acompanhados os eventos que ocorreram em Eglin, Ramey, Natal e Cachoeira Paulista. Esses eventos sugerem que suas fontes são aurorais, assim como descrito nesse artigo. A atenuação dessas ondas pode ser explicada pelo fato do equador magnético estar funcionando como uma barreira, ou seja, a origem desse fenômeno em regiões aurorais é indicado pela maior amplitude de onda que aparece na zona da aurora de cada hemisfério, enquanto que o seu movimento equatorial é indicado pela atenuação de amplitude da onda em relação ao equador, como explica Horvath e Lovell (2013).

\section{REFERÊNCIAS}

Afraimovich, E. L., Kosogorov, E. A., Leonovich, L. A., Palamartchouk, K. S., Perevalova, N. P., \& Pirog, O. M. (2000). Determining parameters of large-scale traveling ionospheric disturbances of auroral origin using GPS-arrays. Journal of Atmospheric and Solar-Terrestrial Physics, 62(7), 553-565. 
Afraimovich, E. L., Voeykov, S. V., Perevalova, N. P., \& Ratovsky, K. G. (2008). Large-scale traveling ionospheric disturbances of auroral origin according to the data of the GPS network and ionosondes. Advances in Space Research, 42(7), 1213-1217.

Beer, T. (1974). Atmospheric waves. New York, Halsted Press; London, Adam Hilger, Ltd., 1974. $315 \mathrm{p}$.

Bishop, R. L., Aponte, N., Earle, G. D., Sulzer, M., Larsen, M. F., \& Peng, G. S. (2006). Arecibo observations of ionospheric perturbations associated with the passage of Tropical Storm Odette. Journal of Geophysical Research: Space Physics, 111(A11).

Blumen, W. (1968). On the stability of quasi-geostrophic flow. Journal of the Atmospheric Sciences, 25(5), 929-931.

Bowman, G. G. (1965). Travelling disturbances associated with ionospheric storms. Journal of Atmospheric and Terrestrial Physics, 27(11-12), 1247-1261.

Cândido, C. M. N. (2008) Estudos de irregularidades no plasma da camada ionosferica f equatorial e de baixas latitudes no setor longitudinal brasileiro. São José dos Campos,. 283p. Tese de Doutorado em Geofísica Espacial, Divisão de Geofísica Espacial, Instituto Nacional de Pesquisas Espaciais.

Cazuza, E. P. (2014). Estudos de distúrbios ionosféricos propagantes de larga escala (LSTID'S) na região equatorial durante grandes tempestades magnéticas: estudo de casos(Master's thesis, Universidade Federal do Rio Grande do Norte).

Dal Lago, A., Vieira, L. E. A., Echer, E., Gonzalez, W. D., Clúa de Gonzalez, A. L., Guarnieri, F. L., ... \& Schuch, N. J. (2004). Great geomagnetic storms in the rise and maximum of solar cycle 23. Brazilian journal of physics, 34(4B), 1542-1546.

Dal Poz, W. R., \& de Oliveira Camargo, P. (2006). Conseqüências de uma tempestade geomagnética no posicionamento relativo com receptores GPS de simples freqüência. Boletim de Ciências Geodésicas, 12(2).

Fagundes, P. R., Klausner, V., Sahai, Y., Pillat, V. G., Becker-Guedes, F., Bertoni, F. C. P., ... \& Abalde, J. R. (2007). Observations of daytime F2-layer stratification under the southern crest of the equatorial ionization anomaly region. Journal of Geophysical Research: Space Physics, 112(A4).

Fedrizzi, M. (2003). Estudo do efeito das tempestades magnéticas sobre a ionosfera utilizando dados do GPS. São José dos Campos,. 223p. Tese de Doutorado em Geofísica Espacial, Divisão de Geofísica Espacial, Instituto Nacional de Pesquisas Espaciais.

Francis, S. H. (1973). Lower-atmospheric gravity modes and their relation to medium-scale traveling ionospheric disturbances. Journal of Geophysical Research, 78(34), 8289-8295.

Francis, S. H. (1975). Global propagation of atmospheric gravity waves: A review. Journal of Atmospheric and terrestrial Physics, 37(6-7), 1011-1054.

Fuselier, S. A., Trattner, K. J., \& Petrinec, S. M. (2000). Cusp observations of high-and low-latitude reconnection for northward interplanetary magnetic field. Journal of Geophysical Research: Space Physics, 105(A1), 253-266.

Guo, J., Liu, H., Feng, X., Wan, W., Deng, Y., \& Liu, C. (2014). Constructive interference of largescale gravity waves excited by interplanetary shock on 29 October 2003: CHAMP observation. Journal of Geophysical Research: Space Physics, 119(8), 6846-6851. 
Hajkowicz, L. A. (1990). A global study of large scale travelling ionospheric disturbances (TIDs) following a step-like onset of auroral substorms in both hemispheres. Planetary and Space Science, 38(7), 913-923.

Hajkowicz, L. A. (1991). Global onset and propagation of large-scale travelling ionospheric disturbances as a result of the great storm of 13 March 1989. Planetary and Space Science, 39(4), 583-593.

Hargreaves, J. K. (1992). The solar-terrestrial environment: an introduction to geospace-the science of the terrestrial upper atmosphere, ionosphere, and magnetosphere. Cambridge University Press.

Hawlitschka, S. (2006). Travelling ionospheric disturbances (TIDs) and tides observed by a superresolution HF direction finding system. Journal of atmospheric and solar-terrestrial physics, 68(3-5), 568-577.

Hedin, A. E. (1987). MSIS-86 thermospheric model. Journal of Geophysical Research: Space Physics, 92(A5), 4649-4662.

Hines, C. O. (1960). Internal atmospheric gravity waves at ionospheric heights. Canadian Journal of Physics, 38(11), 1441-1481.

Hines, C. O. (1974). Internal atmospheric gravity waves at ionospheric heights. The Upper Atmosphere in Motion, 248-328.

Hoffmann, P., Jacobi, C., \& Borries, C. (2012). Possible planetary wave coupling between the stratosphere and ionosphere by gravity wave modulation. Journal of Atmospheric and SolarTerrestrial Physics, 75, 71-80.

Horvath, I., \& Lovell, B. C. (2013). Equatorial westward electrojet impacting equatorial ionization anomaly development during the 6 April 2000 superstorm. Journal of Geophysical Research: Space Physics, 118(11), 7398-7409.

Hunsucker, R. D. (1982). Atmospheric gravity waves generated in the high-latitude ionosphere: A review. Reviews of Geophysics, 20(2), 293-315.

Huttunen, K. E. J., Koskinen, H. E., Pulkkinen, T. I., Pulkkinen, A., Palmroth, M., Reeves, E. G. D., \& Singer, H. J. (2002). April 2000 magnetic storm: Solar wind driver and magnetospheric response. Journal of Geophysical Research: Space Physics, 107(A12).

Idrus, I. I., Abdullah, M., Hasbi, A. M., Husin, A., \& Yatim, B. (2013). Large-scale traveling ionospheric disturbances observed using GPS receivers over high-latitude and equatorial regions. Journal of Atmospheric and Solar-Terrestrial Physics, 102, 321-328.

Kafle, D. N. (2009). Rayleigh-lidar observations of mesospheric gravity wave activity above Logan, Utah. Utah State University.

Lynn, K. J. W., Gardiner-Garden, R., Sjarifudin, M., Terkildsen, M., Shi, J., \& Harris, T. J. (2008). Large-scale travelling atmospheric disturbances in the night ionosphere during the solarterrestrial event of 23 May 2002. Journal of Atmospheric and Solar-Terrestrial Physics, 70(17), 2184-2195.

MacDougall, J., Abdu, M. A., Batista, I., Buriti, R., Medeiros, A. F., Jayachandran, P. T., \& Borba, G. (2011). Spaced transmitter measurements of medium scale traveling ionospheric disturbances near the equator. Geophysical Research Letters, 38(16). 
Martyn, D. F. (1950). Cellular atmospheric waves in the ionosphere and troposphere. Proc. Roy. Soc. A, 201, 216-234.

Mayr, H. G., Harris, I., Herrero, F. A., Spencer, N. W., Varosi, F., \& Pesnell, W. D. (1990). Thermospheric gravity waves: Observations and interpretation using the transfer function model (TFM). Space Science Reviews, 54(3-4), 297-375.

McLandress, C. (1998). On the importance of gravity waves in the middle atmosphere and their parameterization in general circulation models. Journal of Atmospheric and Solar-Terrestrial Physics, 60(14), 1357-1383.

Ngwira, C. M., McKinnell, L. A., Cilliers, P. J., \& Yizengaw, E. (2012). An investigation of ionospheric disturbances over South Africa during the magnetic storm on 15 May 2005. Advances in Space Research, 49(2), 327-335.

Nicolls, M. J., Kelley, M. C., Coster, A. J., González, S. A., \& Makela, J. J. (2004). Imaging the structure of a large-scale TID using ISR and TEC data. Geophysical Research Letters, 31(9).

Nogueira, P. A. B. (2009). Estudo da anomalia de ionização equatorial e dos ventos termosféricos meridionais durante períodos calmos e perturbados na região de baixas latitudes brasileira.

Oliveira, A. L. P., Cazuza, E. P., Neto, J. Q. M., da Silva Junior, J. P., Barbosa, A. A. X., Borba, G. L. \& de Lima Alcântara, M. (2016). Ondas de gravidade na estratosfera equatorial brasileira: estudo de caso. HOLOS, 8, 24-35.

Rees, D. (1995). Observations and modelling of ionospheric and thermospheric disturbances during major geomagnetic storms: A review. Journal of Atmospheric and Terrestrial Physics, 57(12), 1433-1457.

Rieger, M., \& Leitinger, R. (2002). The effect of travelling ionospheric disturbances (TIDs) on GNSS user systems. Proc. 27th General Assembly of URSI, Maastricht, The Netherlands.

Rishbeth, H., \& Garriott, O. K. (1969). Introduction to ionospheric physics. IEEE Transactions on Image Processing.

Shiokawa, K., Otsuka, Y., Ogawa, T., Balan, N., Igarashi, K., Ridley, A. J., ... \& Yumoto, K. (2002). A large-scale traveling ionospheric disturbance during the magnetic storm of 15 September 1999. Journal of Geophysical Research: Space Physics, 107(A6).

Silva Júnior, J. P. D. (2015). Desenvolvimento de um magnetômetro fluxgate para estudos de clima espacial e aeroespacial (Master's thesis, Universidade Federal do Rio Grande do Norte).

Sobral, J. H. A., Abdu, M. A., \& Sahai, Y. (1985). Equatorial plasma bubble eastward velocity characteristics from scanning airglow photometer measurements over Cachoeira Paulista. Journal of atmospheric and terrestrial physics, 47(8-10), 895-900.

Stepanov, A. E., Filippov, L. D., \& Sofronov, S. K. (2012). Trave-ling ionospheric disturbances: data of meridional chain of ionosondes and model calculations. In Proc. of the 9th Intern. Conf. «Problems of Geocosmos». SPb. (p. 405).

Sterling, D. L., Hooke, W. H., \& Cohen, R. (1971). Traveling ionospheric disturbances observed at the magnetic equator. Journal of Geophysical Research, 76(16), 3777-3782.

Testud, J. (1970). Gravity waves generated during magnetic substorms. Journal of Atmospheric and Terrestrial Physics, 32(11), 1793-1805.

Thome, G. (1968). Long-period waves generated in the polar ionosphere during the onset of magnetic storms. Journal of Geophysical Research, 73(19), 6319-6336. 
Tsuda, T., Nishida, M., Rocken, C., \& Ware, R. H. (2000). A global morphology of gravity wave activity in the stratosphere revealed by the GPS occultation data (GPS/MET). Journal of Geophysical Research: Atmospheres, 105(D6), 7257-7273.

Vargas, F. A. (2007). Investigação do fluxo de momento das ondas de gravidade na alta atmosfera através da aeroluminescência. Instituto Nacional de Pesquisas Espaciais, São José dos Campos, Brasil.

Vlasov, A., Kauristie, K., van de Kamp, M., Luntama, J. P., \& Pogoreltsev, A. (2011, November). A study of traveling ionospheric disturbances and atmospheric gravity waves using EISCAT Svalbard Radar IPY-data. In Annales Geophysicae(Vol. 29, No. 11, p. 2101). Copernicus GmbH.

Wrasse, C. M. (2004). Estudo de geração e propagação de ondas de gravidade atmosféricas. Instituto Nacional de Pesquisas Espaciais, São José dos Campos, Brasil.

Yamashita, C. S. (1999). Efeito das tempestades magnéticas intensas na ionosfera de baixa latitude. São José dos Campos. 75p. Dissertação de Mestrado em Geofísica Espacial, Instituto Nacional de Pesquisas Espaciais.

Yiğit, E., Knižová, P. K., Georgieva, K., \& Ward, W. (2016). A review of vertical coupling in the Atmosphere-Ionosphere system: Effects of waves, sudden stratospheric warmings, space weather, and of solar activity. Journal of Atmospheric and Solar-Terrestrial Physics, 141, 1-12. 